Development of CCD cameras for soft $\mathrm{X}$-ray imaging at the National Ignition Facility

N. E. Palmer, A. T. Teruya, M. B. Schneider, P. M. Bell

August 22, 2013

SPIE Conference

San Diego, CA, United States

August 25, 2013 through August 29, 2013 
This document was prepared as an account of work sponsored by an agency of the United States government. Neither the United States government nor Lawrence Livermore National Security, LLC, nor any of their employees makes any warranty, expressed or implied, or assumes any legal liability or responsibility for the accuracy, completeness, or usefulness of any information, apparatus, product, or process disclosed, or represents that its use would not infringe privately owned rights. Reference herein to any specific commercial product, process, or service by trade name, trademark, manufacturer, or otherwise does not necessarily constitute or imply its endorsement, recommendation, or favoring by the United States government or Lawrence Livermore National Security, LLC. The views and opinions of authors expressed herein do not necessarily state or reflect those of the United States government or Lawrence Livermore National Security, LLC, and shall not be used for advertising or product endorsement purposes. 


\section{Development of CCD cameras for soft X-ray imaging at the National Ignition Facility}

Target Diagnostics Physics and Engineering for Inertial Confinement Fusion II/SPIE Optical Engineering + Applications August 27, 2013

N. E. Palmer, A. T. Teruya, M. B. Schneider, P. M. Bell

\section{$\| \leftarrow$ Lawrence Livermore National Laboratory}

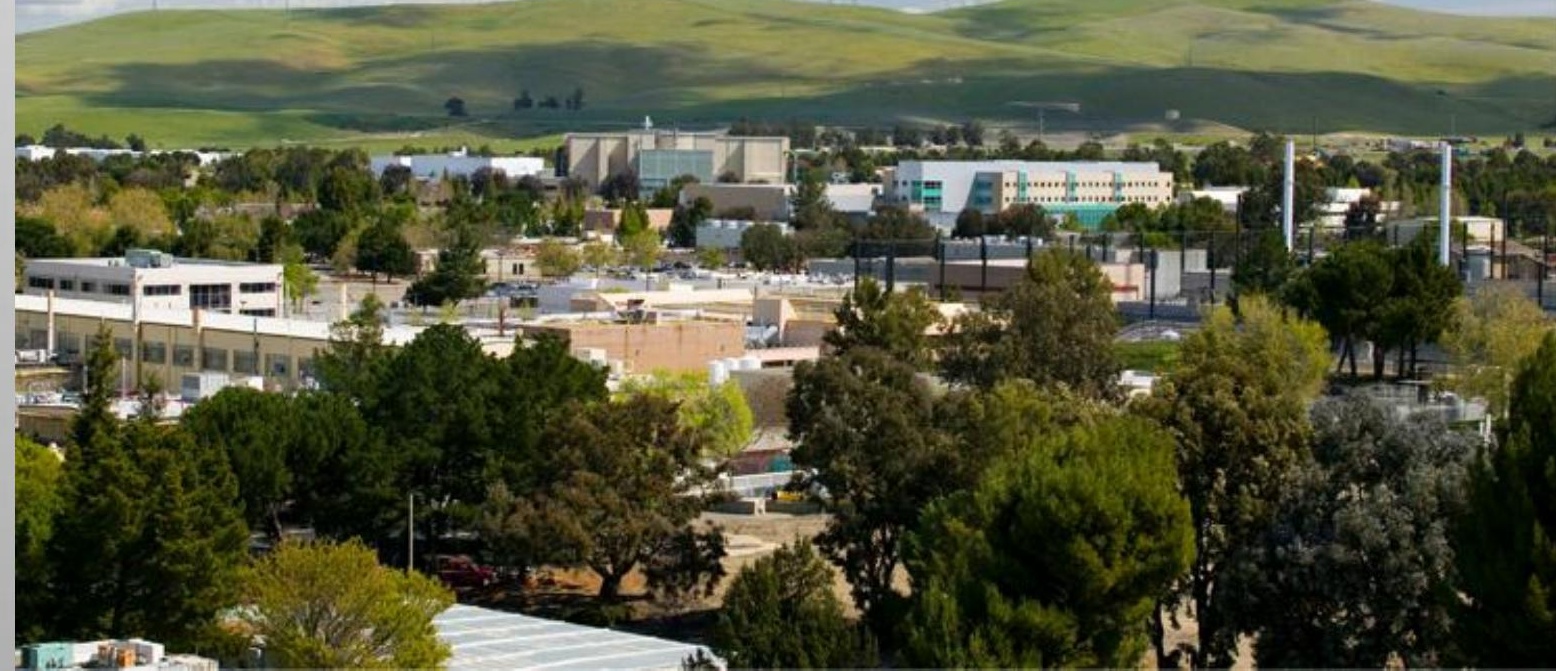




\section{List of Collaborators}

- LLNL

- Alan Teruya, Nathan Palmer, Perry Bell, Marilyn Schneider

- National Security Technologies, Inc. (NSTec)

- Michael Haugh, Ed Romano, Ken Jacoby, Michael Charest

- Spectral Instruments

- Gary Sims, Kevin Toerne, Ken Rodenberg

- University of Arizona, Imaging Technology Laboratory

- Michael Lesser 


\section{Outline}

- Introduction to the Static X-Ray Imager (SXI)

- Requirements for a replacement charge-coupled device (CCD) camera for SXI

- Testing of a prototype camera and procurement of replacement CCD cameras

- Performance

- Replacement camera with a front-illuminated CCD sensor

- Replacement camera with a back-illuminated CCD sensor

- Future use in SXI 


\section{SXI provides X-ray pinhole images of the top and bottom of targets}

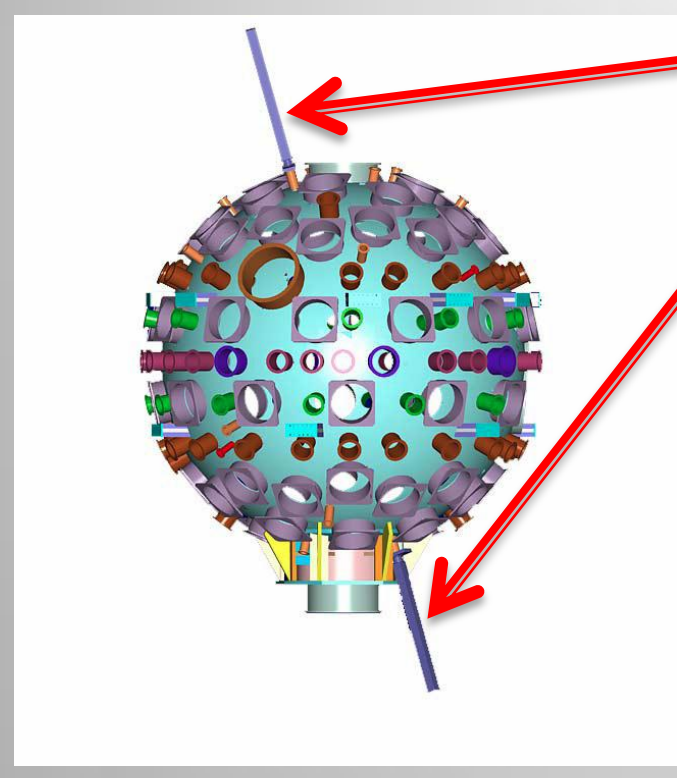

- X-Ray pinhole images acquired using a CCD camera

- Image plate substituted on higher yield shots
- SXI-Upper located at port 018-123

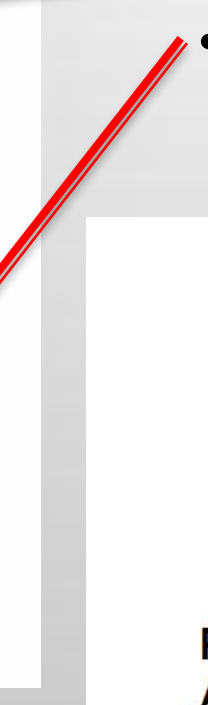

Gate Valvel

Chamber Wall

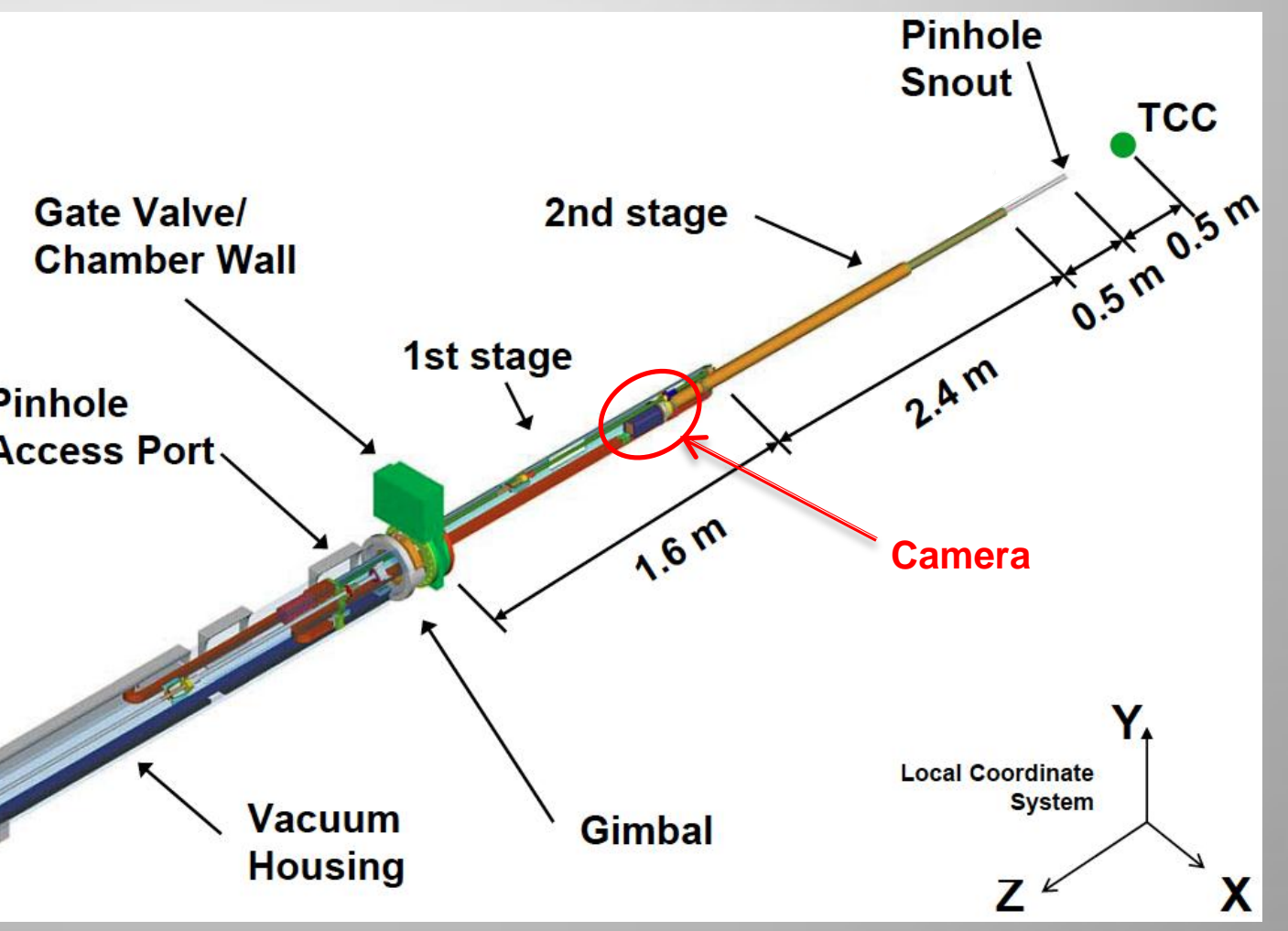




\section{Dynamic range is extended by varied filtering of multiple pinholes}

- SXI measures the clear aperture of the laser entrance hole (LEH) of the hohlraum
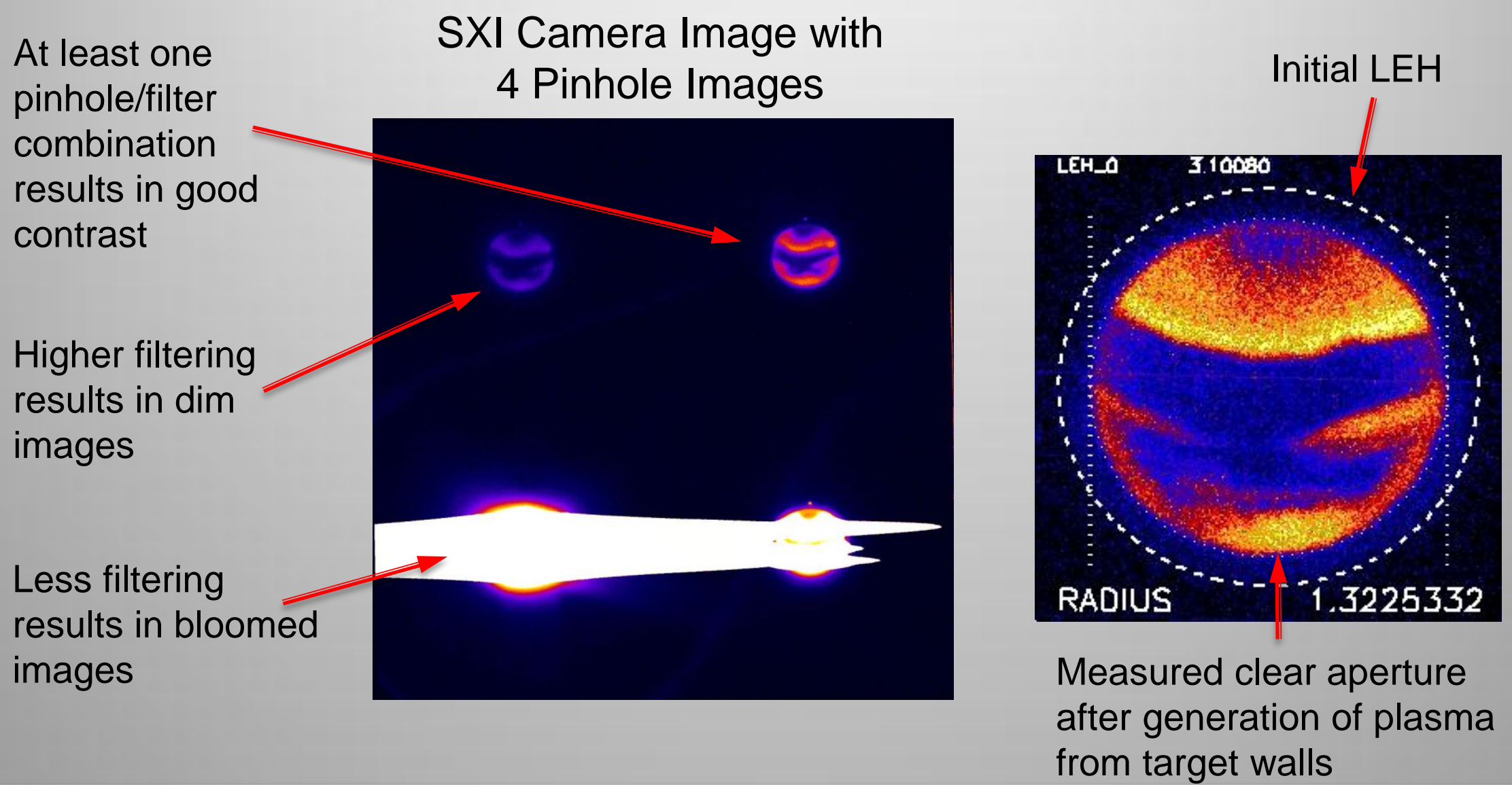


\section{SXI-Upper includes a soft X-ray mirror channel}

- $10^{\circ}$ angle-of-incidence tungsten/boron carbide multilayer mirror is designed to reflect a $100 \mathrm{eV}$ band around $870 \mathrm{eV}$
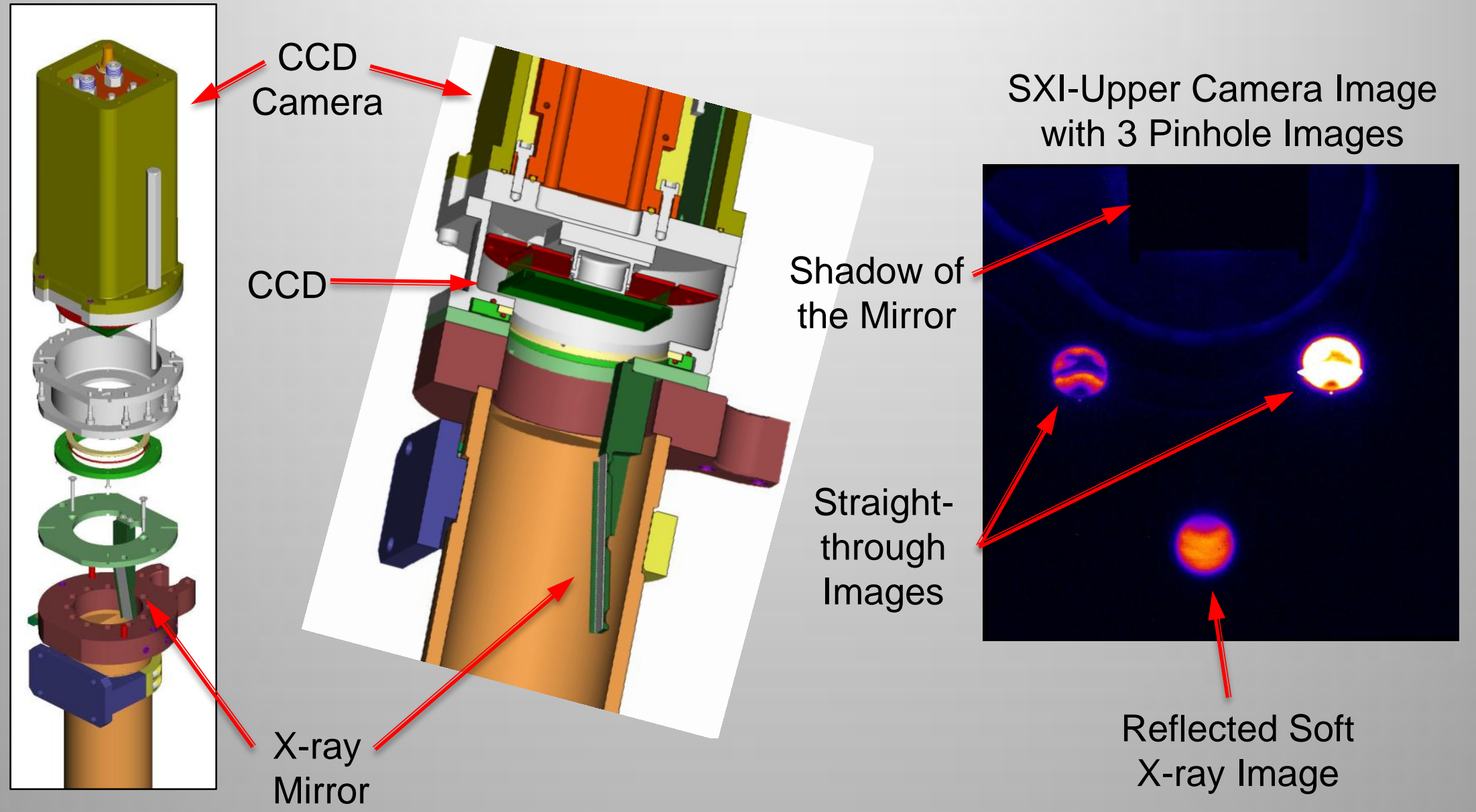


\section{The current SXI camera records X-rays in the range of 700 to $8,000 \mathrm{eV}$}

- Modified Spectral Instruments SI-800 in an LLNL-designed vacuum-immersible airbox housing

- CCD: Scientific Imaging Technologies, Inc. (SITe) 2,048 x 2,048, $49 \mathrm{~mm}$ x 49mm, 24 um pixel, back illuminated
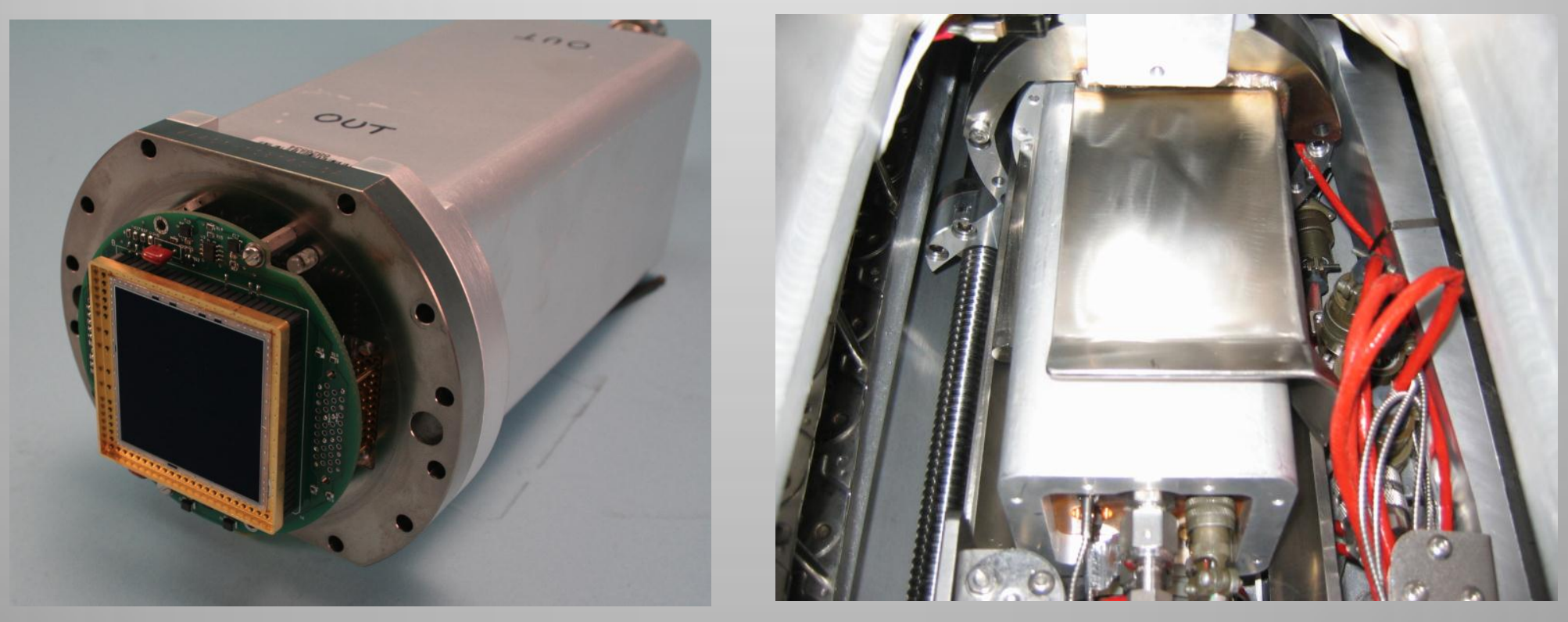


\section{SXI had a need for new cameras}

- Only three existing cameras - One spare for two diagnostics

- The CCD sensor is out of production and LLNL has no spares

- The CCD manufacturer (SITe) is long out of business

- The new camera had to be a drop-in replacement - modification to the positioner hardware and utilities is cost prohibitive

- For SXI-Lower:

- X-rays in 3-5 keV range

- Front-illuminated CCDs have acceptable sensitivity

- For SXI-Upper:

- X-rays in 800-900 eV range

- Back-illuminated CCDs required, but none available off the shelf

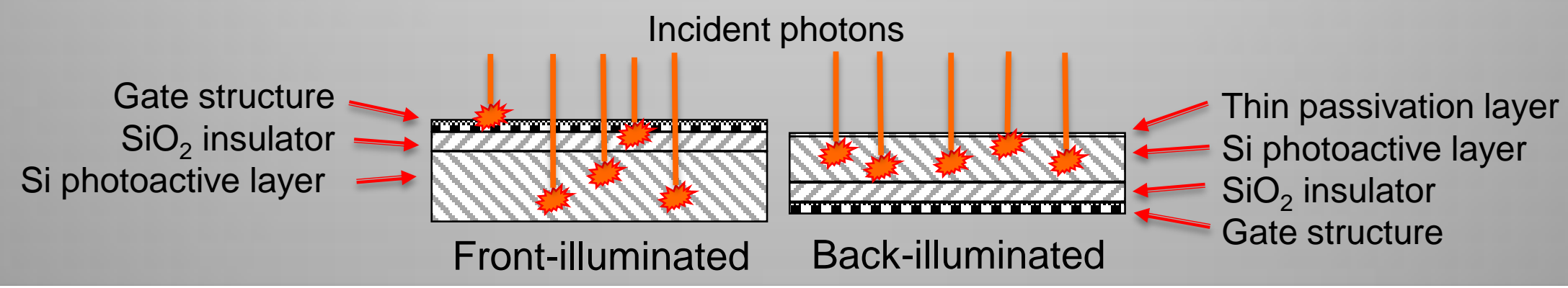




\section{A candidate CCD was identified}

- Teledyne Dalsa IA-DJ-02084 CCD

- 2084 × 2084 pixel, $50.2 \mathrm{~mm} \times 50.2 \mathrm{~mm}, 24$ × 24 um pixels, front illuminated

- Full well capacity 170 ke- (typical), RMS noise 13 e-

- Spectral Instruments built a test camera using the 1044 x 1044 pixel version of the sensor for quantum efficiency (QE) evaluation

- Due to the front illumination structure, the QE was lower than the existing cameras, but was deemed usable in the 2 to $8 \mathrm{keV}$ range if filtering was adjusted

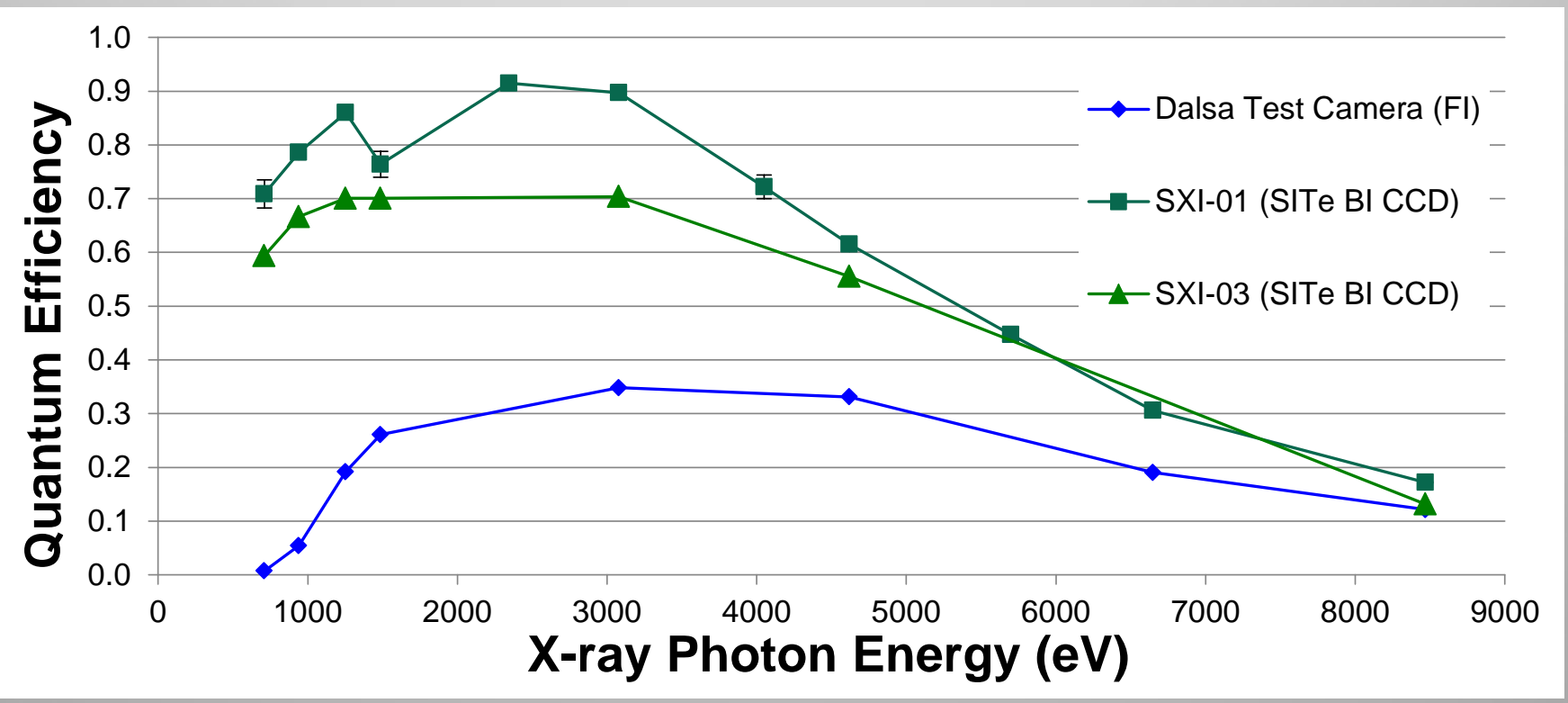


New cameras were ordered for use in the SXIs

- Physical drop-in replacements for existing cameras

- Use gigabit optical fiber link, but same NIF control software

- 16-bit image depth

- Three cameras ordered for SXI-Lower (2 to $8 \mathrm{keV}$ )

- Off-the-shelf front-illuminated Dalsa CCD

- Two cameras ordered for SXI-Upper (700 to 900 $\mathrm{eV})$

- SI purchased nine CCDs on the die from Teledyne Dalsa and contracted with the Imaging Technology Laboratory at the University of Arizona, Tucson, to develop a backthinning process to convert them to back-illuminated

- The process yielded three usable sensors 


\section{Front-illuminated Cameras}

- Spectral Instruments built three cameras (SXI05, -07, -08) with stock Teledyne Dalsa IA-DJ02084 Class I or II grade CCDs

- Standard calibration measurements have been done on SXI-05 by NSTec

- Quantum Efficiency

- Camera Efficiency

- Flat Field

- Linearity and Dynamic Range 


\section{Front-illuminated Camera - QE}

- QE measurements fit well to a model for CCD image sensors developed by Dunn and Steel ${ }^{1}$ using estimates of the thickness of the active detection region $(19 \mu \mathrm{m})$ and the electrode gate structure $(3 \mu \mathrm{m})$

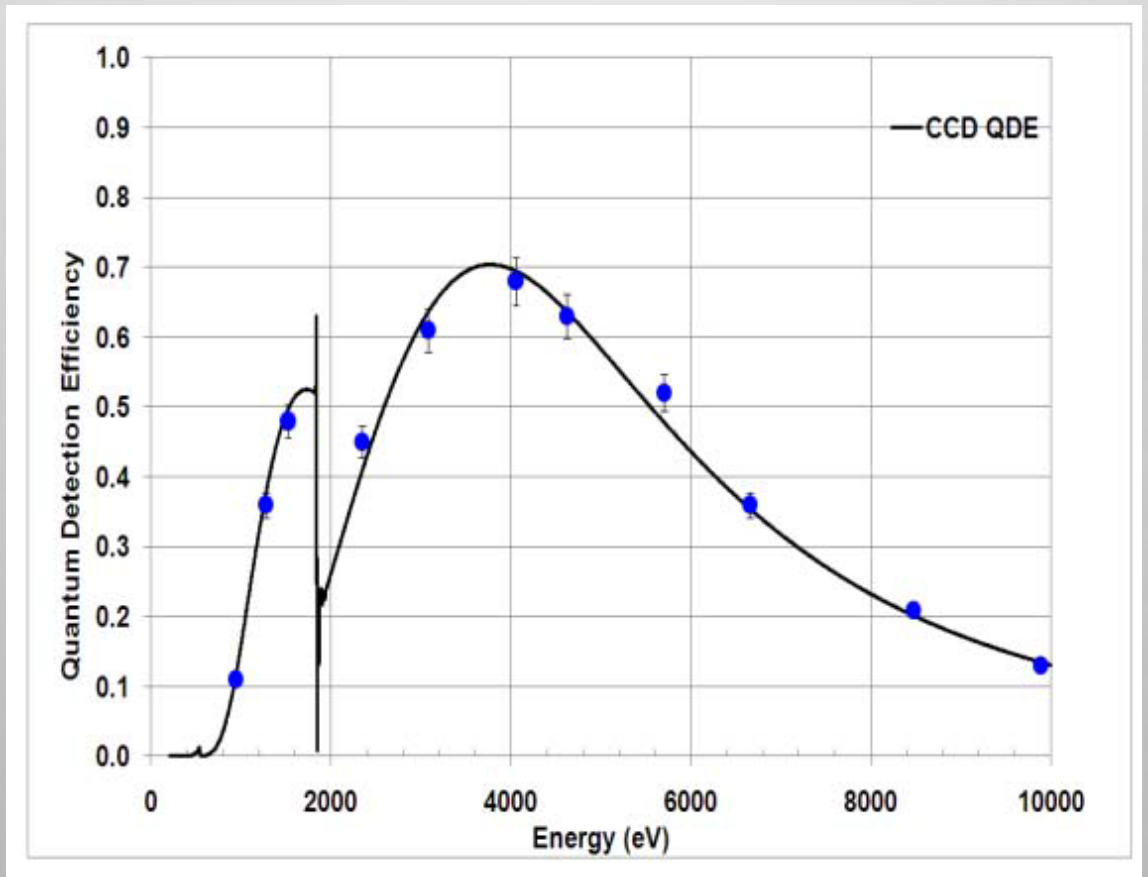

1 J. Dunn, and A. B. Steel, "Absolute determination of charge-coupled device quantum detection efficiency using Si K-edge x-ray absorption fine structure", Rev. Sci. Instrum. 83, 10E120 (2012) 


\section{Front-illuminated Camera - QE Comparison}

- QE of the camera with a front-illuminated CCD is closer to that of the existing cameras than was expected based upon the test camera

- Higher QE above $5 \mathrm{keV}$ because of thicker epitaxial $\mathrm{Si}$

- Lower QE below $2 \mathrm{keV}$ due to electrode gate structure

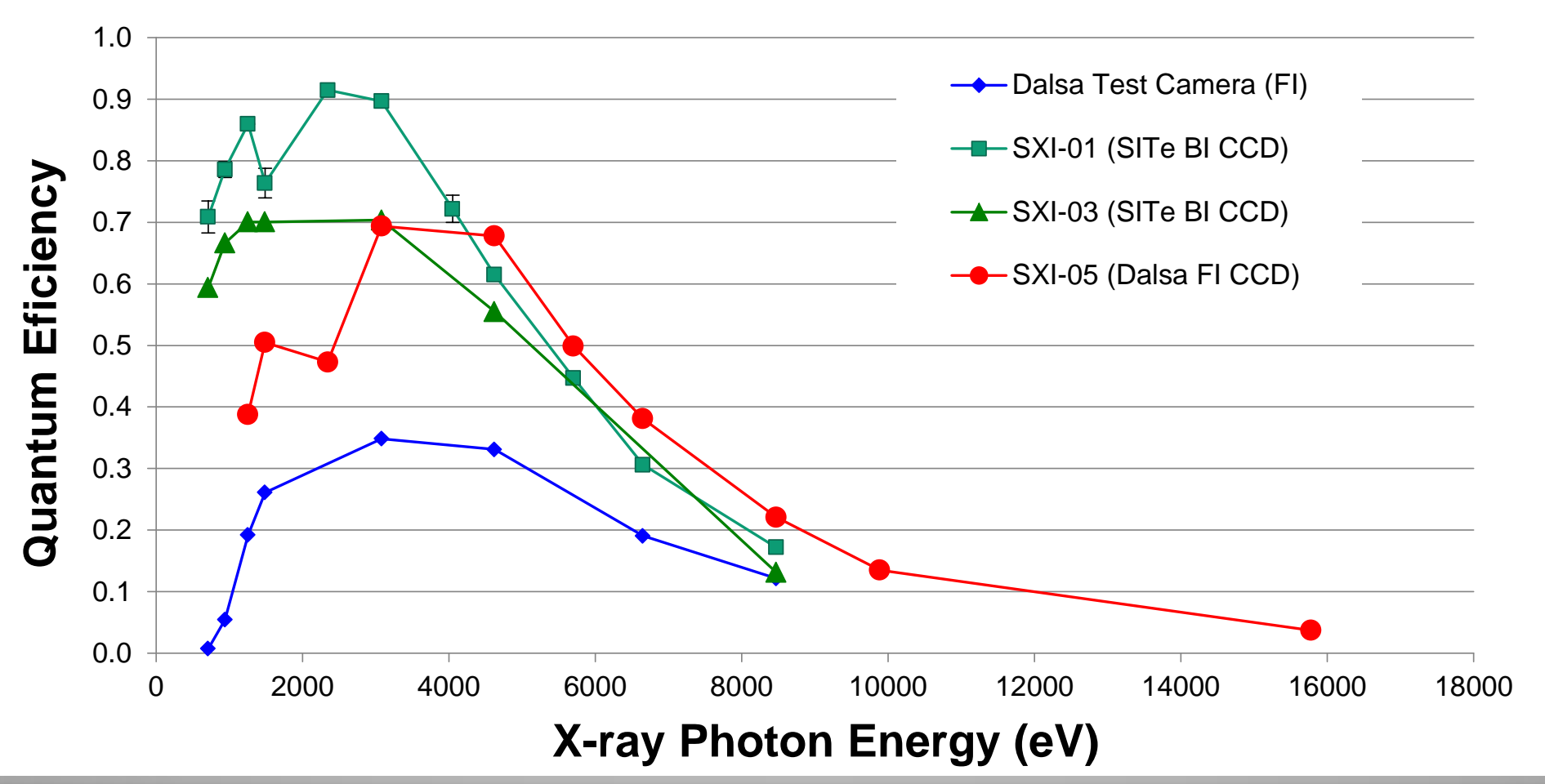




\section{Front-illuminated Camera - Dynamic Range}

- Deviation from linear at $~ 45,000$ counts

- Bias image noise 10 counts

- Dynamic range 4500:1

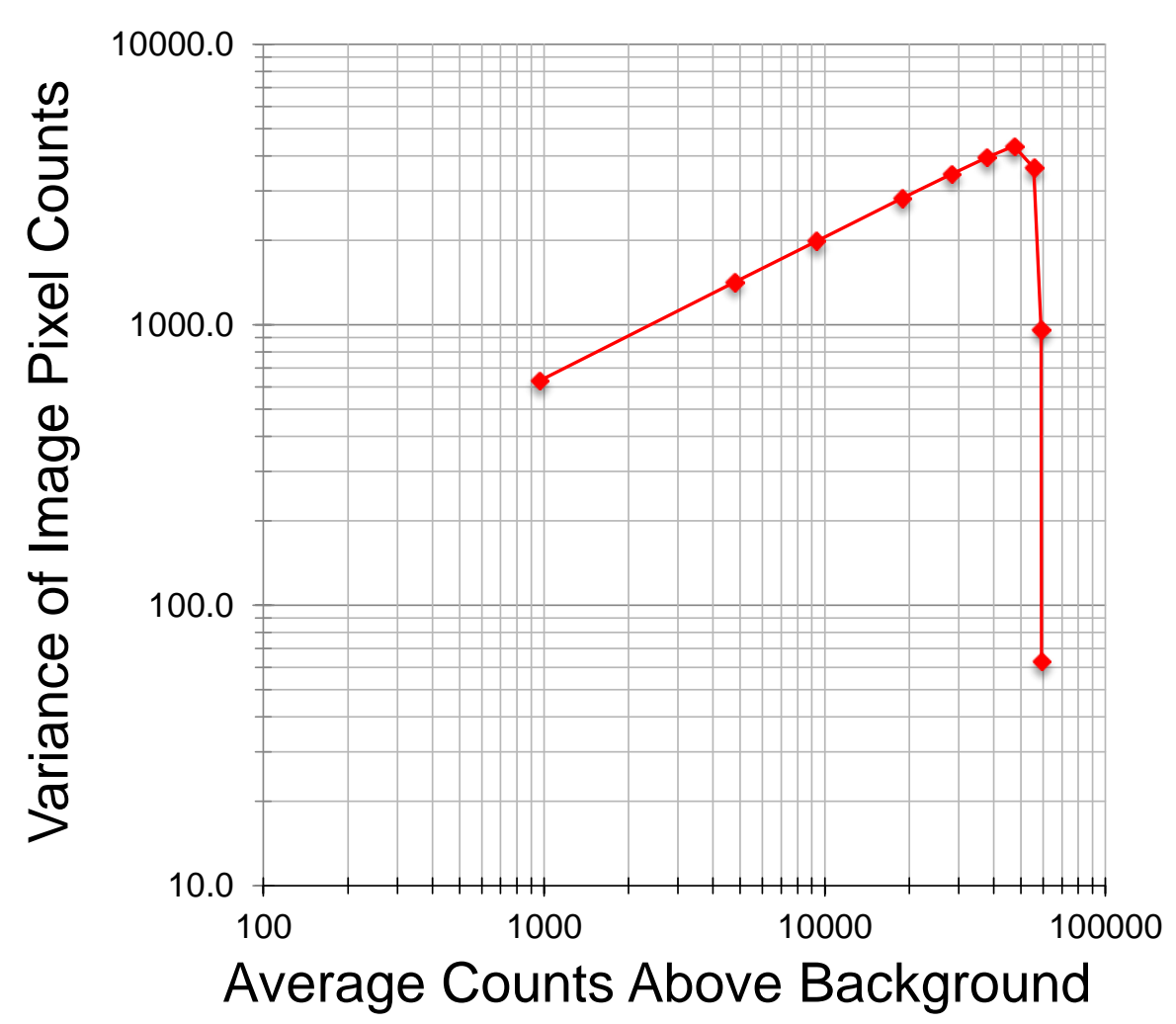




\section{Front-illuminated Camera - Flat Field (8470 eV)}

- Flat field masks show variation across the CCD of only a few percent

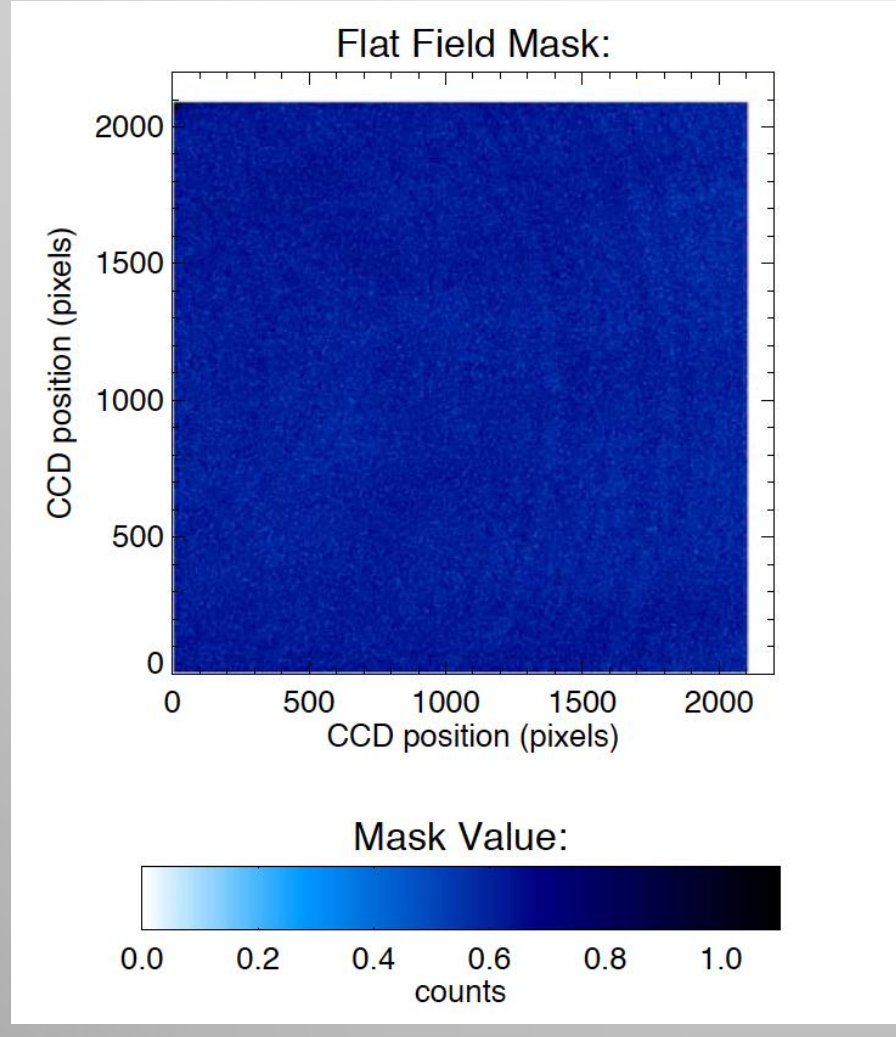

(Uniform illumination from 8470 eV X-ray source)
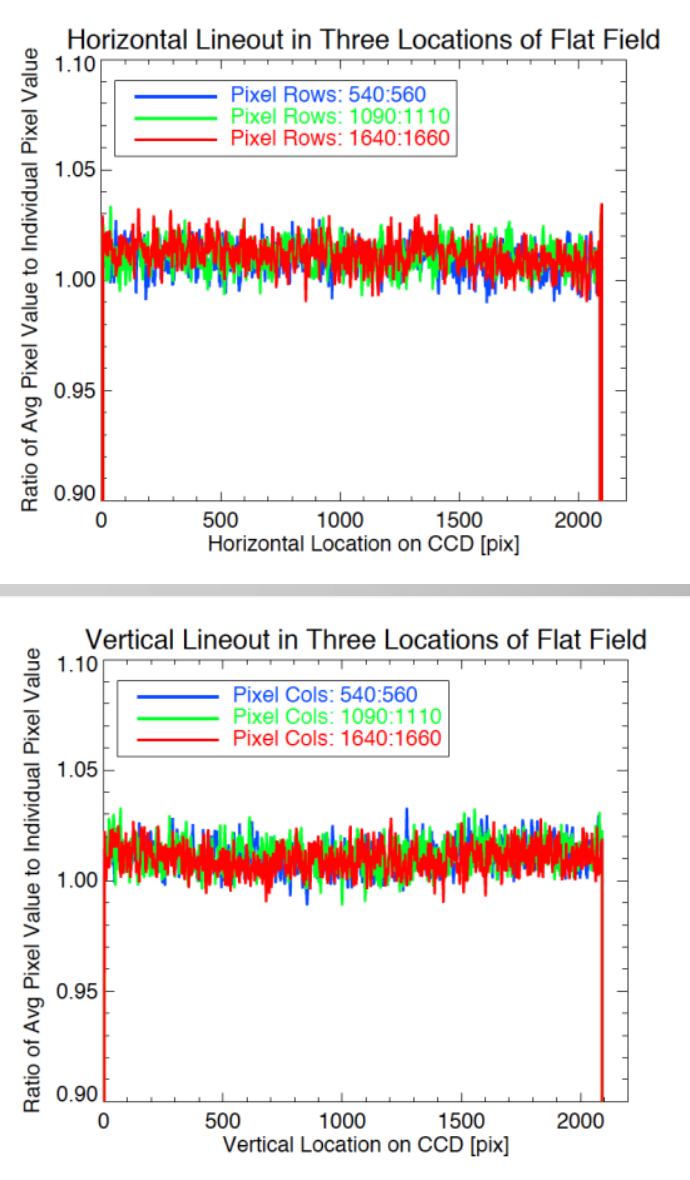


\section{Back-illuminated Cameras}

- Two cameras were built (SXI-04, -06) using two of the three usable back-thinned CCDs

- These cameras exhibit higher read noise and more bad pixels than the front-illuminated cameras

- Longer traces on sensor circuit board to account for reversal of bond pads when sensor was flipped $=>$ More sensitive to power supply noise and other EMI

- Some pixels damaged during the back-thinning process

- QE and flat field measurements have been done - still need to measure dynamic range 


\section{Back-illuminated Camera - QE}

- As expected the QE at lower X-ray energies (below $3 \mathrm{keV}$ ) is higher than the front-illuminated CCD where the gate structure blocks photons

- Above $4 \mathrm{keV}$ the front-illuminated CCD has higher QE due to it having a thicker layer of Si to capture photons

- Performance of the back-illuminated Dalsa CCD camera is similar to the existing SITe CCD cameras

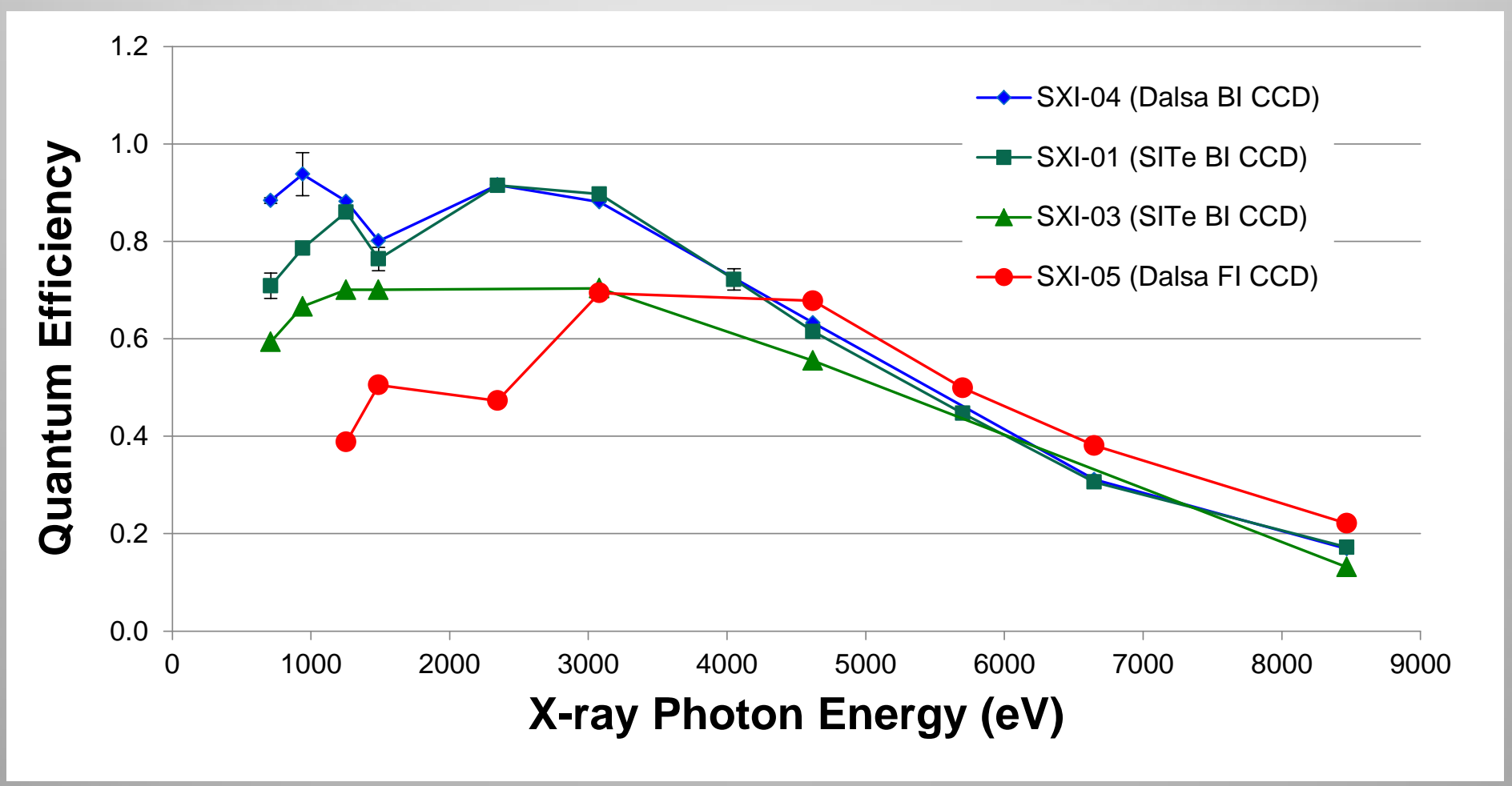




\section{Back-illuminated Camera - Flat Field (1254 eV)}

- At lower energy $(1254 \mathrm{eV})$ any nonuniformity in the passivation layer or any contamination on the surface causes variation in the flat field

- Circular defect in the corner is a less sensitive spot damaged during the etching process. Otherwise, variation is only a few percent.
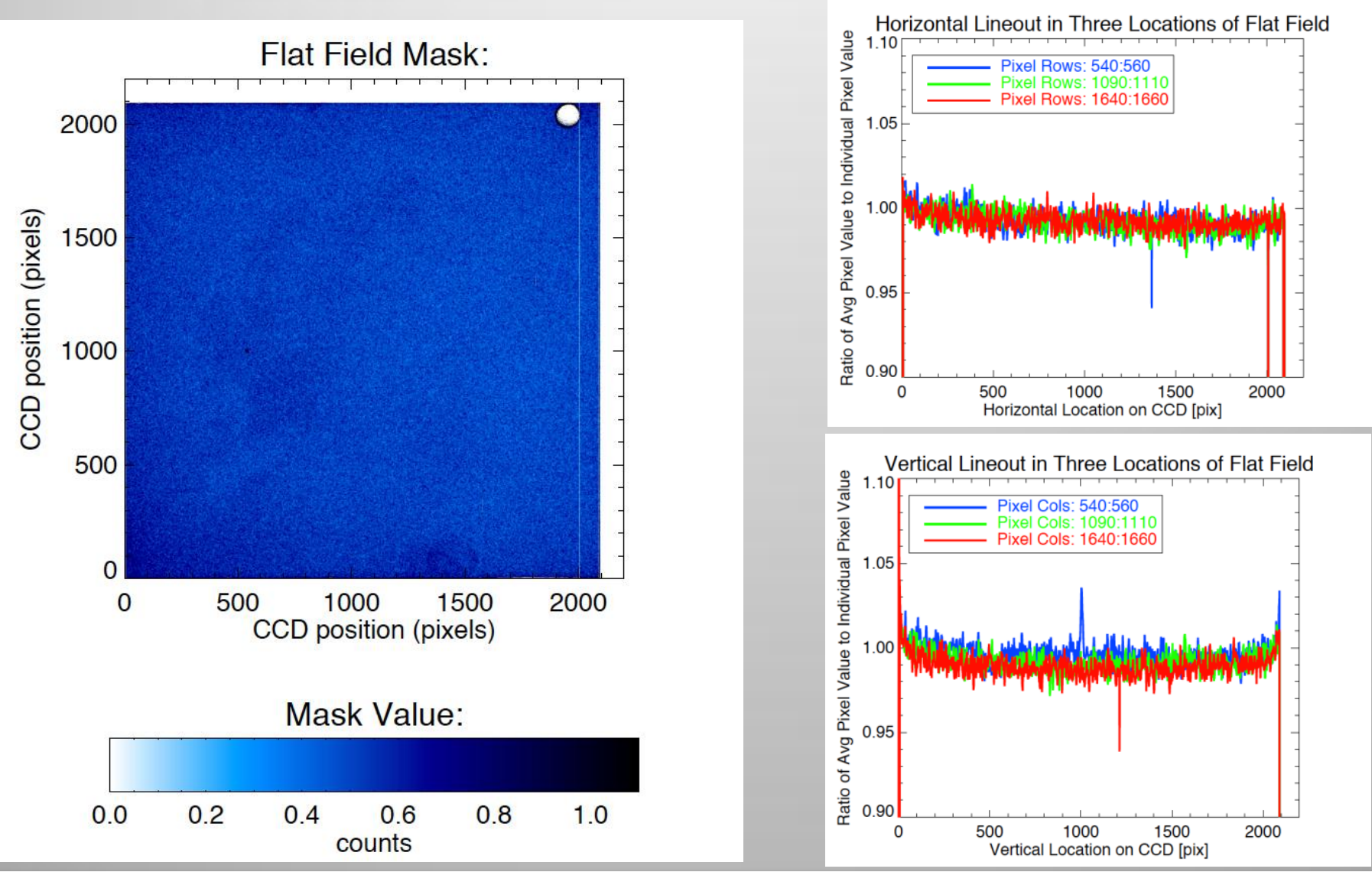


\section{Back-illuminated Camera - Flat Field (8470 eV)}

- At higher energy $(8470 \mathrm{eV})$ the flat field varies due to nonuniformity in the thinning of the Si. Sensor is thicker in the center, thinner around the edges.

- Variation across the sensor as much as $10 \%$
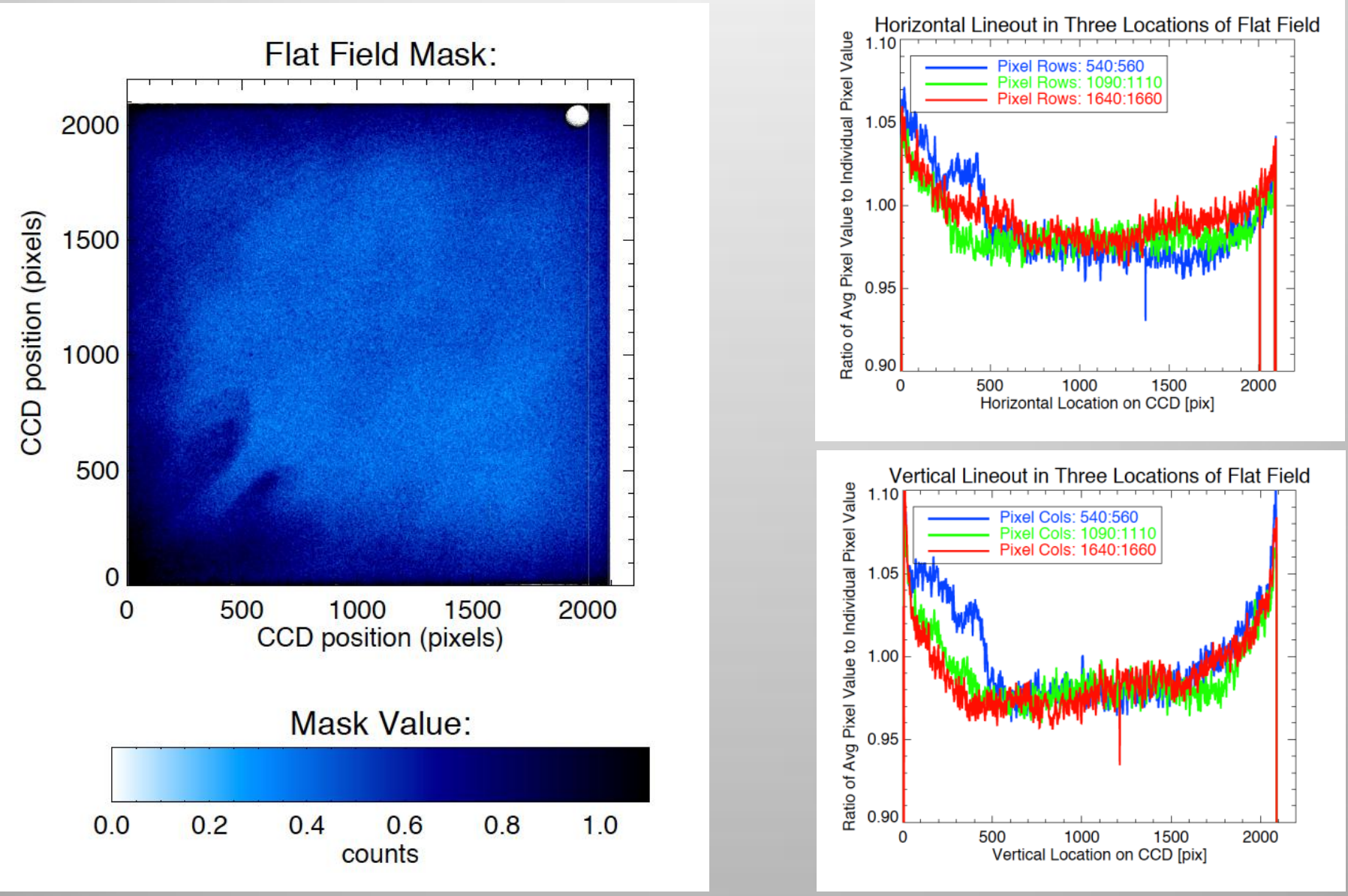
The cameras are suitable for use in SXI and are being prepared for fielding

- The replacement cameras use existing cables and utilities

- 28 VDC Power

- Multimode 62.5/125 $\mu \mathrm{m}$ communication fibers

- Cooling water

- Trigger via coax. cable

- Control computers with upgraded gigabit optical fiber link boards have been installed at NIF

- Cameras are undergoing testing to pass NIF cleanliness standards and require final calibration 
느 Lawrence Livermore 